Pacific Journal of Mathematic 


\title{
A GENERALIZATION OF ATOMIC BOOLEAN ALGEBRAS
}

\author{
R. S. PIERCE
}

1. Introduction. A Boolean algebra $B$ is called atomic if every non-zero element of $B$ contains an atom. A variant of this definition is the equivalence: $B$ is atomic if and only if $B$ contains a dense (i.e., coinitial in $B-\{0\}$ ) subset which is totally unordered. In this paper, we will investigate the properties of Boolean algebras which contain dense subsets of somewhat more general order type than the totally unordered sets.

Definition 1.1. Let $\alpha$ be an infinite cardinal number. A partially ordered set $P$ will be called $\alpha$-compact if $P$ is closed under finite meets, contains a zero element and satisfies the condition that if $M \cong P$ has cardinality $\leqq \alpha$ and no finite subset of $M$ has zero meet, then $M$ has a non-zero lower bound in $P$.

The use of the term "compact" is of course motivated by the topological analogy.

Definition 1.2. A Boolean algebra $B$ will be called $\alpha$-atomic if $B$ contains a dense subset which is $\alpha$-compact.

Since a totally unordered set becomes $\alpha$-compact (for all $\alpha$ ) if a zero element is adjoined to it, an atomic Boolean algebra is $\alpha$-atomic for all cardinals $\alpha$.

The organization of the paper is as follows. Section two is devoted to the construction of examples of $\alpha$-atomic Boolean algebras. In section three, some properties of $\alpha$-atomic Boolean algebras are proved. Section four presents a representation theorem for $\alpha$-atomic algebras.

Throughout the paper, $\alpha$ will denote a fixed infinite cardinal number. The abbreviation $\alpha$-B.A. will be used for $\alpha$-complete Boolean algebra. The terms $\alpha$-subalgebra, $\alpha$-ideal, $\alpha$-homorphism, $\alpha$-field, etc. have their usual meanings. Thus, an $\alpha$-homorphism of an $\alpha$-B.A. is a homomorphism preserving $\alpha$-joins; an $\alpha$-subalgebra of an $\alpha$-B.A. is a subalgebra closed under formation of $\alpha$-joins in the enveloping algebra. It is sometimes convenient to use the symbol $\infty$ in place of $\alpha$ with the meaning that the corresponding property is to hold for all cardinals.

The lattice operations of join, meet and complement are designated by $\mathrm{v}, \Lambda$, and (') respectively. The symbols 0 and $u$ denote the zero and unit in a Boolean algebra. Set operations are indicated by rounded symbols: $\cap, \cup$ and $\subseteq$ stand for intersection, union and inclusion

Received July 24, 1957. 
respectively. The empty set is denoted by $Q$. The symbol $|A|$ represents the cardinality of the set $A$. For any cardinal number $\alpha$, the smallest cardinal greater than $\alpha$ is denoted $\alpha^{+}$.

2. Examples. We have already observed that any atomic Boolean algebra is $\infty$-atomic. The converse is also true.

Theorem 2.1. A Boolean algebra is o-atomic if and only if it is atomic.

Proof. Let $B$ be $\infty$-atomic. If $|B|=\alpha$, it is possible to find a dense subset $D$ in $B$ which is $\alpha$-complete. Let $M$ be a maximal dual ideal in $D$. Then $M$ has the finite meet property and $|M| \leqq \alpha$. Hence, $M$ has a non-zero lower bound $a$ in $D$. By the maximality of $M$, it is clear that $a$ is an atom of $B$. Since, by Zorn's lemma, every non-zero element of $D$ is contained in a maximal dual ideal of $D$, it follows that every element of $D$ contains an atom. But $D$ is dense in $B$ so every element of $B$ contains an atom. Thus $B$ is atomic.

In order to construct an $\alpha$-atomic B.A., it is enough to exhibit an $\alpha$-compact partially ordered set $P$ which is disjunctive, that is, satisfies the condition that for any $a \not b b$, there exists $c \in P$ such that $0 \neq c \leqq a$ and $c \wedge b=0$. Indeed, any disjunctive partially ordered set can be imbedded as a dense subset in a complete Boolean algebra (see [1]), and if the partially ordered set is $\alpha$-compact, then the B.A. will necessarily be $\alpha$-atomic. This complete B.A. is determined up to isomorphism by the disjunctive partially ordered set. In fact, a more precise statement is true.

Lemma 2.2. Let $B_{1}$ and $B_{2}$ be complete Boolean algebras. Let $P_{1}$ and $P_{2}$ be dense subsets of $B_{1}$ and $B_{2}$ which are closed under meets. Suppose $\varphi$ is an isomorphism of $P_{1}$ on $P_{2}$. Then $\varphi$ has a unique extension to an isomorphism of $B_{1}$ on $B_{2}$.

This result is proved in [1] for example.

We describe a fairly general method of constructing partially ordered sets which are disjunctive and $\alpha$-compact.

Let $I$ be a non-empty index set. Let $\left\{X_{i} \mid i \in I\right\}$ be a collection of sets, each containing at least two elements. Put $X=\prod_{i \in I} X_{i}$. Suppose $\mathfrak{M}$ is a given non-empty collection of subsets of $I$ with the properties

(a) $\mathfrak{M}$ is closed under finite unions,

(b) $\mathfrak{M}$ is $\alpha$-directed : any subcollection of $\mathfrak{M}$ with cardinality $\leqq \alpha$ has an upper bound in $\mathfrak{M}$.

Let $M \in \mathfrak{M}$ and $\varphi \in \prod_{i \in M} X_{i}$, that is, $\varphi$ is a function on $M$ such that $\varphi(i) \in X_{i}$ for all $i \in M$. Denote 


$$
A_{M, \varphi}=\left\{\chi \in X|\chi|_{M}=\varphi\right\} .
$$

Finally, let $\Omega=\Omega\left(I, X_{i}, \mathfrak{M}\right)$ be the collection of all $A_{M, \varphi}$, together with the empty set.

Lemma 2.3. The set $\Omega$, ordered by inclusion, is an $\alpha$-compact, disjunctive partially ordered set.

Proof. Observe that $A_{M, \varphi} \cong A_{N, \psi}$ if and only if $M \supseteqq N$ and $\left.\varphi\right|_{N}=\psi$. On the other hand, $A_{M, \varphi} \cap A_{N, \psi}=Q$ if and only if there exists $i \in M \cap N$ such that $\varphi(i) \neq \psi(i)$. The fact that $\Omega$ is meet closed and disjunctive is a routine consequence of these observations and property (a) of $\mathfrak{M}$. The fact that $\Omega$ is $\alpha$-directed follows from property (b) of $\mathfrak{M}$.

A special case of this contruction is particularly interesting. If $I$ is arbitrary, each $X_{i}$ is a two element set and $\mathfrak{M}$ consists of all subsets $A$ of $I$ with $|A| \leqq \alpha$, then the conditions for the application of Lemma 2.3 are fulfilled. The $\alpha$-compact partially ordered set $\Omega$ in this case is completely determined by the cardinal numbers $\alpha$ and $\beta=|I|$. Thus we can designate this $\Omega$ simply as $\Phi_{\alpha \beta}$. Let $B$ be a complete Boolean algebra containing $\Phi_{\alpha \beta}$ as a dense subset and define $F_{\alpha \beta}$ to be the smallest $\alpha$-subalgebra of $B$ containing $\Phi_{\alpha \beta}$. It is clear from 2.2 that $F_{\alpha \beta}$ is determined up to isomorphism by $\Phi_{\alpha \beta}$.

Theorem 2.4. The Boolean algebra $F_{\alpha \beta}$ is isomorphic to the $\alpha$-field of subsets of $X$ generated by $\Phi_{c_{\beta}}$ and is a free $\alpha$-representable algebra with $\beta$ generators.

Remark. A Boolean algebra is called $\alpha$-representable if it is the $\alpha$-homomorphic image of an $\alpha$-field (see [2]). The fact that the class of all such algebras is equationally definable and therefore admits free algebras has been investigated in [5]. Indeed, Sikorski proves in [7] that the $\alpha$-field generated by $\Phi_{\alpha \beta}$ is a free $\alpha$-representable algebra with $\beta$ generators. Thus it is only necessary to prove the first assertion of 2.4 .

Proof. Let $B_{1}$ be the $\alpha$-field (in $X$ ) generated by $\Phi_{\alpha_{\beta}}$. Note that $\Phi_{\alpha \beta}$ is closed under $\alpha$-intersections and that the complement of any set of $\Phi_{\alpha \beta}$ is a union of sets of $\Phi_{\alpha \beta}$. Hence, by Lemma 5.2 of [4] (quoted in (4.3) below), $\Phi_{\alpha \beta}$ is dense in $B_{1}$. It follows from 2.2 that $B_{1}$ is isomorphic to $F_{\alpha \beta}$.

COROLlaRY 2.5. Every $\alpha$-representable Boolean algebra is an $\alpha$-homomorph of an $\alpha$-atomic, $\alpha$-field.

It is easy to see that if $\beta \leqq \alpha$, then $\Phi_{\alpha \beta}$ is atomic and hence so is 
any Boolean algebra containing $\Phi_{\alpha \beta}$ as a dense subset. However, if $\beta>\alpha$, then a Boolean algebra $B$ containing $\Phi_{\alpha \beta}$ as a dense subset is not even $\alpha^{+}$-atomic. This is a consequence (by (3.3) below) of the stronger result that $B$ is not $\alpha^{+}$-distributive (see [9]).

Theorem 2.6. Let $B$ be a Boolean algebra containing $\Phi_{\alpha \beta}$ as a dense subset. Suppose also that $\beta>\alpha$. Then $B$ is not $\alpha^{+}$-distributive.

Proof. Let $J \leqq I$ have cardinality $\alpha^{+}$. For $i \in I$, denote $a_{i k}=$ $\left\{\chi \in X \mid \chi(i)=x_{i k}\right\}(k=1,2)$, where $X_{i}=\left\{x_{i 1}, x_{i 2}\right\}$. Then $a_{i 1} \vee a_{i 2}=u$ (since no element of $\Phi_{\alpha \beta}$ is disjoint from both $a_{i 1}$ and $a_{i 2}$ ). But if $M \in \mathfrak{M}$, then $J \nsubseteq M$, so there exists $j \in J-M$. For this index, $\bigcap_{i \in M} a_{i, \varphi(i)} \nsubseteq a_{j k}$ $(k=1,2)$ for all $\varphi \in 2^{M}$. Thus

$$
\bigwedge_{i \in J}\left(a_{i 1} \vee a_{i \Xi}\right)=u>0=\bigvee_{\varphi}\left(\Lambda_{i \in J} a_{i, \varphi(i)}\right),
$$

so $B$ is not $\alpha^{+}$-distributive.

REMARK. The referee has pointed out that 2.6 is related to the results in Scott's paper [6]. Scott constructs a Boolean algebra $B_{x}$ (for each regular cardinal $\alpha$ ) which, when $\alpha=\beta^{+}$, is equivalent to the completion of $F_{\beta \beta^{+}}$.

3. Properties of $\alpha$-atomic algebras. The term "covering" will be used to designate a subset of a Boolean algebra whose least upper bound is the unit element.

Lemma 3.1. Let $B$ be an $\alpha$-complete, $\alpha$-atomic Boolean algebra. Then $B$ has the following property:

$\left(^{*}\right)$ if $\left\{A_{\sigma} \mid \sigma \in S\right\}$ is a family of coverings of $B$ such that $|S| \leqq \alpha^{+}$ and if $b \neq 0$ in $B$, then there is a choice function $\varphi$ on $S$ such that $\varphi(\sigma) \in A_{\sigma}$ with the property that if $T \leqq S$ and $|T| \leqq \alpha$, then

$$
b \wedge \Lambda_{\sigma \in T} \varphi(\sigma) \neq 0 \text {. }
$$

Proof. Let $T \subseteq B$ be dense and $\alpha$-compact. Denote by $\lambda$ the least ordinal of cardinality $\alpha^{+}$. We can assume that $S$ consists of the ordinals $\sigma<\lambda$. By transfinite induction, define functions $f: S \rightarrow T$ and $\varphi$ on $S$ with $\varphi(\sigma) \in A_{\sigma}$ having properties

(i) $\sigma<\tau$ implies $0<f(\tau) \leqq f(\sigma) \leqq b$,

(ii) $f(\sigma) \leqq \varphi(\sigma)$.

These are constructed in the following way. Assume $f(\sigma)$ has been defined for all $\sigma<\tau$, where $\tau<\lambda$. By $\alpha$-compactness, $c=\Lambda_{\sigma<\tau} f(\sigma) \neq 0$. If $\tau=1$, then $c=u$. Let $\varphi(1) \in A_{1}$ satisfy $\varphi(1) \wedge b \neq 0$. Such an element 
exists, since $b=b \wedge u=b \wedge \bigvee A_{1}=\bigvee\left\{b \wedge a \mid a \in A_{1}\right\}$. Let $f(1) \in T$ be chosen arbitrarily, satisfying $0 \neq f(1) \leqq \varphi(1) \wedge b$. If $\tau>1$, then $c \leqq b$. Choose $\varphi(\tau) \in A_{\tau}$ so that $\varphi(\tau) \wedge c \neq 0$. As before, some element of $A_{r}$ will satisfy this requirement. Using the fact that $T$ is dense, it is possible to find $f(\tau) \in T$ such that $0<f(\tau) \leqq \varphi(\tau) \wedge c$. With this construction, it is clear that (i) and (ii) are fulfilled.

Now if $T \leqq S$ and $|T| \leqq \alpha$, then since $\lambda$ is regular, there exists $\eta<\lambda$ such that $\tau<\eta$ for all $\tau \in T$. Hence,

$$
b \wedge \Lambda_{\sigma \in T} \varphi(\sigma) \geqq b \wedge \Lambda_{\tau<\eta} \varphi(\tau) \geqq b \wedge \Lambda_{\tau<\eta} f(\tau) \geqq f(\eta)>0 .
$$

This is the required conclusion.

Corollary 3.2. Any $\alpha^{+}$-complete, $\alpha$-atomic B.A. is $\alpha^{+}$-representable.

Proof. It is easy to see that the condition $\left(^{*}\right)$ of $(3.2)$ implies Smith's property $\left(P_{\alpha+}\right)$ (see [8]). Hence, (3.2) follows from Theorem 4.1 of [8].

Corollary 3.3. Any $\alpha$-complete, $\alpha$-atomic B.A. is $(\alpha, \infty)$-distributive.

For the definition of $(\alpha, \infty)$-distributivity, the reader is referred to [9] or [4]. The property $\left({ }^{*}\right)$, together with (2.3) of [4] implies (3.3).

If $B$ is a complete B.A. containing $\Phi_{\alpha \beta}$ as a dense subset, then $B$ is $\alpha^{+}$-representable by (3.2). If $\beta>\alpha$, then $B$ is $(\alpha, \infty)$-distributive, but not $\alpha^{+}$-distributive by (2.6). Hence, $B$ is not $2^{\alpha+}$-representable. If we admit the generalized continuum hypothesis, this means that $B$ is not $\gamma$-representable for any $\gamma>\alpha^{+}$, which partially answers a question raised by Chang [2; p. 213].

In [3], the author conjectures that any $\alpha$-distributive $2^{\alpha}$-complete B.A. is $2^{\alpha}$-representable. This conjecture now appears rather unlikely in view of Theorem 3.4 of [8], since its validity, together with the generalized continuum hypothesis, would imply a positive answer to Souslin's problem. However, for an $\alpha$-subalgebra $B^{\prime}$ of an $\alpha$-atomic Boolean algebra $B$, it is true that $2^{\alpha}$-completeness implies $\alpha^{+}$-representability. For $B^{\prime}$ is also an $\alpha$-subalgebra of $\bar{B}$, the normal completion of $B$. But $\bar{B}$ is $\alpha^{+}$-representable and $\alpha$-distributive by (3.2) and (3.3). Therefore, to see that $B^{\prime}$ is $\alpha+$-representable, we have only to notice that it must actually be an $\alpha+$-subalgebra of $\bar{B}$.

THEOREM 3.4. Let $\bar{B}$ be an $\alpha$-distributive, $2^{\alpha}$-complete Boolean algebra. Suppose $B$ is an $\alpha$-subalgebra of $\bar{B}$ which is $2^{\alpha}$-complete. Then $B$ is a $2^{v}$-subalgebra of $\bar{B}$.

REMARK. This theorem is well known for fields of sets. (See [9], 
Theorem 3.10 and the references given there.) We will give a proof, since the result seems to have been overlooked in [3] and [9].

Proof. Let $Q \subseteq B$ and $|Q| \leqq 2^{\infty}$. Let $b=$ l.u.b. $Q$ in $B$ and $\bar{b}=$ l.u.b. $Q$ in $\bar{B}$. Then $b \geqq \bar{b}$. Assume $b>\bar{b}$ and set $\bar{c}=b \wedge \bar{b}^{\prime}$. Let $B_{1}$ be the $\alpha$-distributive Boolean algebra: $\{\bar{d} \in \bar{B} \mid \bar{d} \leqq \bar{c}\}$. Denote by $\bar{h}$ the mapping $\bar{B} \rightarrow B_{1}$ given by $\bar{h}(\bar{\alpha})=\bar{a} \wedge \bar{c}$. Evidently $\bar{h}$ is a complete homorphism of $\bar{B}$ and hence the restriction $h$ of $\bar{h}$ to $B$ is an $\alpha$-homomorphism. The image $h(B)$ is an $\alpha$-subalgebra of $B_{1}$ and therefore is $\alpha$-distributive. By Theorem 3.6 of [9] or (6.5) of [3], $h$ is a $2^{x}$-homomorphism. But obviously $Q$ is contained in the kernel of $h$. Thus, $h(b)=1 . u \cdot b_{{ }_{B}}\{h(a) \mid a \in Q\}=0$. But $\bar{c} \leqq b$, so $\bar{c}=u \wedge \bar{c}=\bar{h}(u)=\bar{h}(b \vee \bar{c})=$ $\bar{h}(b) \vee \bar{h}\left(\bar{c}^{\prime}\right)=h(b) \vee\left(\bar{c}_{\Lambda}^{\prime} \bar{c}\right)=0$, a contradiction. Thus, $b=\bar{b}$, which is the desired conclusion.

CoRollary 3.5. Let $\beta$ be weakly attainable from the infinite cardinal $\alpha$. Suppose $\bar{B}$ is a $\beta$-distributive, $2^{\beta}$-complete B.A. and $B$ is an $\alpha$-subalgebra of $\bar{B}$ which is $2^{\beta}$-complete. Then $B$ is a $2^{\beta}$-subalgebra of $\bar{B}$.

Proof. Clearly, if $\xi$ is a singular cardinal and $B$ is an $\eta$-subalgebra of $\bar{B}$ for all $\eta<\xi$, then $B$ is a $\xi$-subalgebra. Using this fact, 3.5 follows from 3.4 by transfinite induction.

4. The representation theorems. Not every $\alpha$-atomic B.A. is an $\alpha$-field, since the normal completion of an atomless $\alpha$-field will not, in general, be an $\alpha$-field ( $\alpha$ being infinite). However, we will prove that every $\alpha$-atomic algebra is a dense subalgebra of the normal completion of an $\alpha$-field. Of course, since any B.A. is a dense subalgebra of its normal completion, it suffices to prove that any complete, $\alpha$-atomic B.A. contains a dense subalgebra $B_{0}$ which is isomorphic to an $\alpha$-field.

Lemma 4.1. Let be an $\alpha$-complete, $\alpha$-atomic B.A. Then $B$ contains a dense, $\alpha$-compact subset which is closed under $\alpha$-meets.

Proof. By Definition 1.2, $B$ contains a dense $\alpha$-compact subset $T$. The set of all $\alpha$-meets of elements of $T$ will clearly be a dense, $\alpha$-compact subset of $B$ which is closed under $\alpha$-meets.

LEMMA 4.2. Let $F$ be a disjunctive, $\alpha$-compact partially ordered set which is closed under $\alpha$-meets. Let $X$ be the set of all proper maximal dual ideals of $F$. For $a \in F$, let $\varphi(a)=\{P \in X \mid a \in P\}$. Then $\varphi$ is an $\alpha$-meet preserving, order isomorphism of $F$ into the Boolean algebra of 
all subsets of $X$. The image $f$ of $\varphi$ has the property that the complement of any set of $f$ is a union of sets of $f$.

Proof. Since $F$ is $\alpha$-compact and closed under $\alpha$-meets, every maximal dual ideal of $F$ is also closed under $\alpha$-meets. Hence, $\varphi$ preserves $\alpha$-meets by the usual argument.

If $c \neq 0$ in $F$, then $\varphi(c) \neq Q$, since every non-zero element is contained in a proper maximal dual ideal. Since $F$ is disjunctive, $a \not \subset b$ implies the existence of $c \in F$ with $0 \neq c \leqq a$ and $b \wedge c=0$. Hence, $Q \neq \varphi(c) \leqq \varphi(a)$ and $\varphi(b) \cap \varphi(c)=Q$. Therefore, $\varphi(a) \varsubsetneqq \varphi(b)$.

If $P \in X-\varphi(a)$, then $a \notin P$. By the maximality of $P$, there exists $b \in P$ such that $a \wedge b=0$. Then $P \in \varphi(b) \subseteq(\varphi(a))^{c}$. This shows that $(\varphi(a))^{c}$ is a union of sets of $f$.

For the proof of the main theorem of this section, we need a known result.

Lemma 4.3. Let $f$ be a family of subsets of a set $X$ with the properties that $f$ is closed under $\alpha$-intersections and the complement of any set of $f$ is a union of sets of $f$. Let $\&$ be the $\alpha$-field generated by $f$. Then $\mathrm{f}$ is dense in $\mathrm{R}$.

The proof of this fact can be found in [4].

TheOREM 4.5. Let $B$ be an $\alpha$-atomic Boolean algebra. Then $B$ is isomorphic to a dense subalgebra of the normal completion of an $\alpha$-atomic $\alpha$-field of sets.

Proof. Let $\bar{B}$ be the normal completion of $B$. Then $\bar{B}$ is $\alpha$-atomic. By (4.1), $\bar{B}$ contains a dense, $\alpha$-compact subset $F$ which is closed under $\alpha$-meets. Since $F$ is dense in the Boolean algebra $\bar{B}, F$ is disjunctive. By (4.2), there is an $\alpha$-isomorphism $\varphi$ of $F$ onto a family $f$ of subsets of a set $X$ with the two properties of (4.3). Let $\&$ be the $\alpha$-field generated by $f$ and let $\Omega_{0}$ be the normal completion of $\Omega$. By (4.3), $f$ is dense in $\&$ and hence in $\Omega_{0}$. Consequently, by (2.2), $\varphi$ extends uniquely to an isomorphism of $\bar{B}$ on $\Omega_{0}$. The restriction of this extension is an isomorphism of $B$ onto a dense sub-algebra of the normal completion of the $\alpha$-atomic $\alpha$-field $\&$.

Corollary 4.6. Any complete, $\alpha$-atomic Boolean algebra is isomorphic to the normal completion of an $\alpha$-atomic $\alpha$-field.

\section{REFERENCES}

1. J. R. Büchi, Die Boolesche Partialordnung, Portugaliae Mathematica 7 (1948), 118-177.

2. C. C. Chang, On the representation of $\alpha$-complete Boolean algebras, Trans. Amer. Math. Soc. 85 (1957), 208-218. 
3. R. S. Pierce, Distributivity in Boolean algebras, Pacific J. Math. 7 (1957), 983-992. 4. R. S. Pierce, Distributivity and the normal completion of Boolean algebras, Pacific J. Math. 8 (1958), 133-140.

5. D. Scott and A. Tarski, Metamathematical proofs of some Boolean representation theorems, in preparation.

6. D. Scott, The independence of certain dislributive laws in Boolean algebras, Trans. Amer. Math. Soc. 84 (1957), 258-261.

7. R. Sikorski, A note to Rieger's paper "On Free $\alpha \xi$-complete Boolean Algebras", Fund. Math. 38 (1951), 53-54.

8. E. C. Smith, Jr., A distributivity condition for Boolean algebras, Ann. of Math. 64 (1956), 551-561.

9. E. C. Smith, Jr. and A. Tarski, Higher degrees of distributivity and completeness in Boolean algebras, Trans. Amer. Math. Soc. 84 (1957), 230-257.

UNIVERSITY OF WASHINGTON 


\section{PACIFIC JOURNAL OF MATHEMATICS}

\section{EDITORS}

\section{David Gilbarg}

Stanford University

Stanford, California

R. A. Beaumont

University of Washington

Seattle 5 , Washington
A. L. Whiteman

University of Southern California

Los Angeles 7, California

L. J. PAIGE

University of California

Los Angeles 24, California

\section{ASSOCIATE EDITORS}
E. F. BECKENBACH
C. E. BURGESS
E. HEWITT
A. HORN

\author{
V. GANAPATHY IYER \\ R. D. JAMES \\ M. S. KNEBELMAN \\ L. NACHBIN
}
I. NIVEN
E. G. STRAUS
T. G. OSTROM
H. L. ROYDEN
G. SZEKERES
M. M. SCHIFFER
F. WOLF
K. YOSIDA

\section{SUPPORTING INSTITUTIONS}

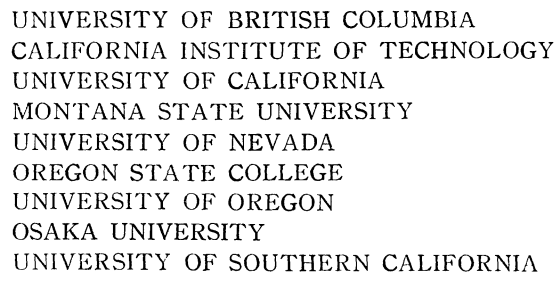

UNIVERSITY OF BRITISH COLUMBIA CALIFORNIA INSTITUTE OF TECHNOLOGY UNIVERSITY OF CALIFORNIA MONTANA STATE UNIVERSITY UNIVERSITY OF NEVADA OREGON STATE COLLEGE UNIVERSITY OF OREGON OSAKA UNIVERSITY UNIVERSITY OF SOUTHERN CALIFORNIA

STANFORD UNIVERSITY

UNIVERSITY OF TOKYO

UNIVERSITY OF UTAH

WASHINGTON STATE COLLEGE

UNIVERSITY OF WASHINGTON

AMERICAN MATHEMATICAL SOCIETY CALIFORNIA RESEARCH CORPORATION HUGHES AIRCRAFT COMPANY SPACE TECHNOLOGY LABORATORIES

Printed in Japan by Kokusai Bunken Insatsusha (International Academic Printing Co., Ltd.), Tokyo, Japan 


\section{Pacific Journal of Mathematics}

\section{Vol. 9, No. 1 \\ May, 1959}

Julius Rubin Blum and Murray Rosenblatt, On the structure of infinitely

divisible distributions . ............................. 1

Robert Geroge Buschman, Asymptotic expressions for

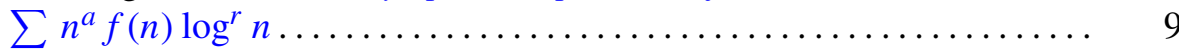

Eckford Cohen, A class of residue systems $(\bmod r)$ and related arithmetical

functions. I. A generalization of Möbius inversion .............. 13

Paul F. Conrad, Non-abelian ordered groups ................... 25

Richard Henry Crowell, On the van Kampen theorem............... 43

Irving Leonard Glicksberg, Convolution semigroups of measures ........ 51

Seymour Goldberg, Linear operators and their conjugates ............ 69

Olof Hanner, Mean play of sums of positional games .............. 81

Erhard Heinz, On one-to-one harmonic mappings ................ 101

John Rolfe Isbell, On finite-dimensional uniform spaces . . ........... 107

Erwin Kreyszig and John Todd, On the radius of univalence of the function

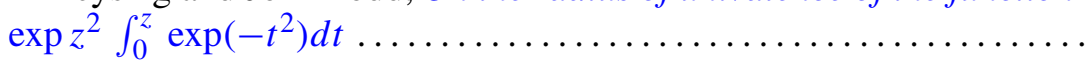

Roger Conant Lyndon, An interpolation theorem in the predicate

calculus......................................... 129

Roger Conant Lyndon, Properties preserved under homomorphism ........ 143

Roger Conant Lyndon, Properties preserved in subdirect products ....... 155

Robert Osserman, A lemma on analytic curves ................ 165

R. S. Phillips, On a theorem due to Sz.-Nagy..................... 169

Richard Scott Pierce, A generalization of atomic Boolean algebras ....... 175

J. B. Roberts, Analytic continuation of meromorphic functions in valued fields................................. 183

Walter Rudin, Idempotent measures on Abelian groups ................ 195

M. Schiffer, Fredholm eigen values of multiply-connected domains ........ 211

V. N. Singh, A note on the computation of Alder's polynomials ......... 271

Maurice Sion, On integration of 1-forms ...................... 277

Elbert A. Walker, Subdirect sums and infinite Abelian groups........... 287

John W. Woll, Homogeneous stochastic processes . .................. 293 